\title{
Acupuncture on Neiguan spot: A promising therapeutic approach for atrial fibrillation by modulating autonomic nerves?
}

\author{
Ling Zhang ${ }^{1 *}$, Zhen Yu Dong ${ }^{2}$, Mei $\mathrm{Ma}^{1}$ and Baopeng Tang ${ }^{1}$ \\ ${ }^{1}$ Hospital of Xinjiang Medical University, China \\ ${ }^{2}$ Xinjiang Medical University, China \\ *Corresponding author: Ling Zhang, Xinjiang Medical University, Urumuqi, Xinjiang, China. \\ To Cite This Article: Ling Zhang. Acupuncture on Neiguan spot: A promising therapeutic approach for atrial fibrillation by modulating \\ autonomic nerves?. Am J Biomed Sci \& Res. 2019 - 2(3). AJBSR.MS.ID.000588. DOI: 10.34297/AJBSR.2019.02.000588
}

Received: April 04, 2019 | Published: April 10, 2019

\section{Opinion}

Atrial fibrillation (AF) is one of the most common arrhythmias with high morbidity and mortality [1]. AF is frequently associated with heart diseases, autonomous nerve imbalance, hypertension, obstructive sleep apnea, inflammation, oxidative stress, and with a high prevalence in the elderly. Previous clinical and experimental researches have confirmed that cardiac autonomic imbalance plays a predominant role in the initiation and maintenance of AF [2-4].

In traditional Chinese medicine, Neiguan acupuncture has been used to treat cardiac arrhythmia and ischemia [5]. Multiple clinical evidences have demonstrated that Neiguan-acupuncture may be an effective approach for AF patients who underwent electrical cardioversion to restore sinus rhythm. The study by Federico Lombardi and his group [6] indicated that acupuncture of Neiguan has unequivocal effects in patients with persistent and paroxysmal $\mathrm{AF}$.

The recurrence rate with acupuncture is similar with oral or intravenous amiodarone on persistent AF patient, but markedly lower than the sham group. In patients with paroxysmal $\mathrm{AF}$, acupuncture significantly reduce the number and duration of episodes of AF. In Chinese researches, meta-analysis of two studies $[7,8]$ showed that for individuals with AF, acupuncture on Neiguan point was shown to be as effective as intravenous amiodarone in conversion from $\mathrm{AF}$ to sinus rate. Another individual study has demonstrated that acupuncture on Neiguan spot plus intravenous deslanoside had better effect on response rate than intravenous deslanoside alone [9]. FAM Jonkmant's case report [10] favored the effectiveness of acupuncture on reducing the recurrence of AF, acupuncture could be a novel approach to treatment resistant AF. But the mechanism behind acupuncture on Neiguan spot needs further elucidation.

Autonomic nerve regulation may be one of the mechanisms of acupuncture on Neiguan spot in the attenuation of AF. Multiple clinical studies have illustrated that acupuncture of Neiguan spot has been shown to lessen nausea and vomiting which are related to vagal modulation, acupuncture of Neiguan spot could be a vagal enhancer in healthy subjects [11]. Joanne W. Y. Chung's meta-analysis [12] supported the possible role of acupuncture on modulating the autonomic she found that LF, HF and LF/HF ratio of HRV significantly decreased. Tatsuo et al. [13] demonstrated that acupuncture on Neiguan spot reduced cardiac excitability and corrected the imbalance between cardiac sympathetic and vagal nerve activity during left stellate ganglion (LSG) stimulation in Yorkshire pigs. Anatomically, Neiguan spot is located on the nerve originating from LSG, the same ganglia that innervate the heart.

Stimulation of Neiguan spot may exert the function of regulating autonomic nerve. Another potential mechanism is about the central nervous system. Acupuncture of Neiguan elicits its cardiac effects by reducing the cardiovascular sympathoexcitatory reflex response. This effect is primarily achieved by inhibiting the rostral ventrolateral medulla to up-regulate inhibitory neurotransmitters such as GABA, opioids, 5-HT, NO and NOS [14].

Acupuncture's anti-AF effect may be related to the stabilizing sympathetic and vagus nerve control mechanisms, rather than direct anti-adrenergic or vagus nerve effects. Although the mechanism is not clear, it does not prevent us from applying it to the clinic. Our team is working on a bracelet that can continuously produce electrical stimulation to the Neiguan spot, which we hope can be used to treat AF. Therefore, we think that acupuncture on Neiguan spot could be a minimally invasive, economic and safe approach to attenuate $\mathrm{AF}$.

\section{References}

1. Xiong Q, Proietti M, Senoo K, Lip GY (2015) Asymptomatic versus symptomatic atrial fibrillation: A systematic review of age/gender differences and cardiovascular outcomes. Int J Cardiol 191: 172-177.

2. Ardell JL (2011) The cardiac neuronal hierarchy and susceptibility to arrhythmias. Heart Rhythm 8(4): 590-591.

3. Kawashima T (2005) The autonomic nervous system of the human heart with special reference to its origin, course, and peripheral distribution. Anat Embryol (Berl) 209(6): 425-438. 
4. Chen PS, Chen LS, Fishbein MC, Lin SF, Nattel S (2014) Role of the autonomic nervous system in atrial fibrillation pathophysiology and therapy. Circ Res 114(9): 1500-1515.

5. Longhurst JC (2007) Electroacupuncture treatment of arrhythmias in myocardial ischemia. Am J Physiol Heart Circ Physiol 292(5): H2032-H2034.

6. Alberto L (2012) Acupuncture for paroxysmal and persistent atrial fibrillation: An effective non-pharmacological tool? World J Cardiol 4(3): 38-49.

7. Chen L, Chen ZHF, Yang XX, Jiang W, Zhou WB, et al. (2012) The efficacy and safety of Neiguan acupoint catgut embedding therapy for atrial fibrillation. J New Chin Med 44: 148-150.

8. Xu HK, Zhang YF (2007) Comparison between therapeutic effects of acupuncture and intravenous injection of amiodarone in the treatment of paroxysmal atrial fibrillation and atrial flutter. Chin J Acupunct Moxibust 27: 96-98.

9. Han BD (2012) Clinical observation on 62 cases of paroxysmal rapid atrial fibrillation treated by acupuncture combined with drug therapy. J Emerg Tradit Chin Med 21: 303-303.
10. Jonkman FAM, Jonkmanbuidin ML (2013) Integrated approach to treatment-resistant atrial fibrillation: additional value of acupuncture. Acupunct Med 31(3): 327-330.

11. Huang ST, Chen GY, Lo HM, Lin JG, Lee YS, et al. (2005) Increase in the vagal modulation by acupuncture at neiguan point in the healthy subjects. Am J Chin Med 33(1): 157-164.

12. Chung JWY, Yan VCM, Zhang H (2014) Effect of acupuncture on heart rate variability: a systematic review. Evidence-based complementary and alternative medicine pp. 83-94.

13. Takamiya T, Kubo Y, Benharash P, Zhou W (2018) Effect of electroacupuncture on porcine cardiac excitability induced by left stellate ganglion stimulation. Auton Neurosci 213: 15-22.

14. Jia L, Jing L, Chen Z, Liang F, Song W, et al. (2012) The influence of PC6 on cardiovascular disorders: a review of central neural mechanisms. Acupunct Med 30(1): 47-50. 